Karger Kompass Pneumol 2017:5:190-194

DOl: 10.1159/00047985

\section{Spektrum Pneumologie - wissenswert, kompakt, anregend}

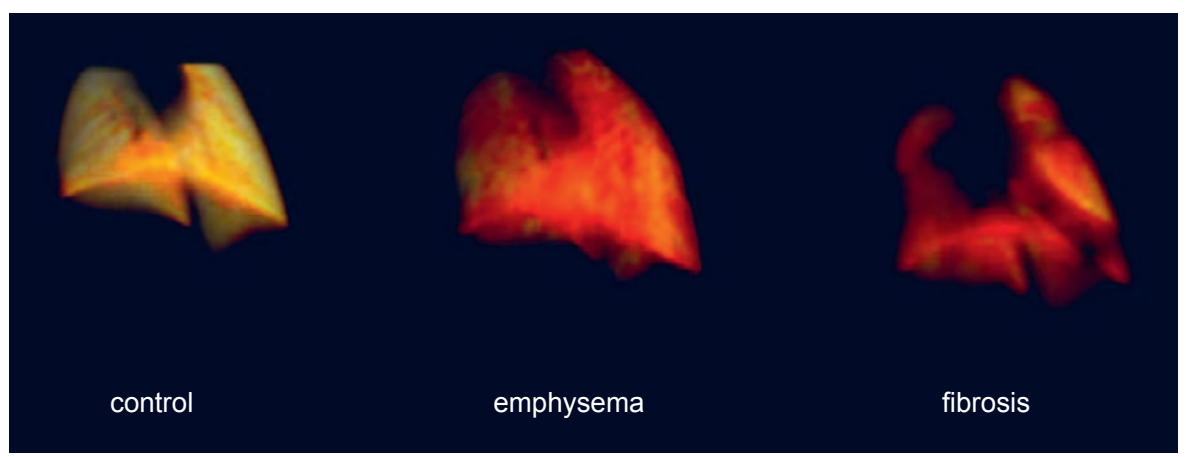

Die Phasenkontrast-Computertomografie an Mausmodellen zeigt, dass Lungenkrankheiten wir COPD (Mitte) oder die Lungenfibrose (Rechts) deutlich besser diagnostisch identifiziert werden können im Vergleich zur herkömmlichen Röntgendiagnostik. (CFranz Pfeiffer / TUM

\section{Deutsche Röntgengesellschaft \\ Ein neuer Blick in die Lunge}

Röntgenologische Untersuchungen erfolgen bisher immer nach dem gleichen Grundprinzip: Röntgenstrahlen werden von unterschiedlichen Geweben unterschiedlich stark absorbiert. Die Strahlen, die nicht absorbiert werden, erreichen den Röntgenfilm (beziehungsweise den digitalen Detektor) und erzeugen dort das Röntgenbild oder die CT-Aufnahme. Die Phasenkontrast-Bildgebung dagegen nutzt die Welleneigenschaften der Röntgenstrahlung. Forscher der Technischen Universität München haben sich dieses Prinzip der Bilderzeugung zunutze gemacht, mit der auch sehr feine Strukturen sowie Strukturen mit ähnlicher Röntgendichte sichtbar werden.

Die Arbeitsgruppe um den Physiker Prof. Dr. Franz Pfeiffer von der TU München verfolgt das Ziel, aus der bisher rein zu Forschungszwecken eingesetzten PhasenkontrastBildgebung eine medizinische Anwendung zu machen. In Kooperation mit dem Klinikum rechts der Isar und der Universität Innsbruck wollen die Forscher die neue Methode jetzt bei Menschen etablieren.

Enormen Nutzen erhoffen sich die Münchner in einem ersten Schritt von der Darstellung der Lunge. Veränderungen der Feinstruktur der Lunge sind für Röntgen- und CT-Untersuchungen bisher weitgehend nicht sichtbar. «Mit der PhasenkontrastBildgebung können wir bis auf die Ebene der Lungenbläschen hinab blicken», erklärt Pfeiffer. «So lassen sich pathologische Veränderungen der Lungenbläschen bei COPD schon in einem sehr frühen Stadium darstellen. Auch Verwachsungen des Lungen- gewebes, wie sie bei einer Lungenfibrose oder auch nach einer Strahlentherapie auftreten können, sind sehr gut erkennbar.» Die Grundidee ist, die neue Technologie als einen zusätzlichen Filter anzubieten, der auf herkömmliche CT-Geräte aufgesetzt werden kann. Die Herstellung dieser speziellen Filter ist anspruchsvoll, denn man benötigt sehr dünne Filter und sehr feinporige Gitterstrukturen, um die Welleneffekte zu erreichen. Zahlreiche große Hersteller von Medizingeräten arbeiten aktuell an entsprechenden Lösungen und bauen Prototypen.

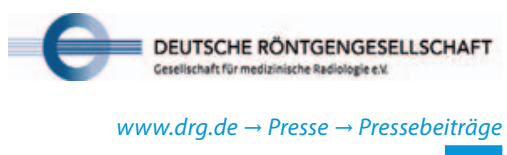

\title{
KARGER
}

(c) 2017 S. Karger GmbH, Freiburg 
In den letzten Jahrzehnten hat die Häufigkeit von Allergie und Asthma in den industrialisierten Ländern massiv zugenommen. Unter den Kindern sind rund 30\% von mindestens einer Allergie betroffen - mit Ausnahme von Kindern, die auf Bauernhöfen aufwachsen. Bei ihnen verläuft die Zunahme der Erkrankungen weniger dramatisch als bei Gleichaltrigen, die zwar im gleichen Dorf, aber nicht auf einem Bauernhof leben. Es ist bekannt, dass Mikroben, die auf Bauernhöfen in größerer Menge und höherer Diversität vorkommen, die Bauernkinder vor Allergien und Asthma schützen. Eine weniger sterile Umgebung wirkt positiv auf die Entwicklung des Immunsystems, da dieses «lernt», aufan sich harmlose Stoffe nicht zu reagieren.

\section{Eine Sialinsäure wirkt als Schutz}

Offenbar haben auch nicht-mikrobielle Stoffe das Potenzial des präventiven Effekts auf das Immunsystem: Das Streicheln von Bauernhoftieren sowie der Schluck Milch direkt ab Hof können ebenfalls Asthma vorbeugen, wie das Forschungsteam rund um Remo Frei

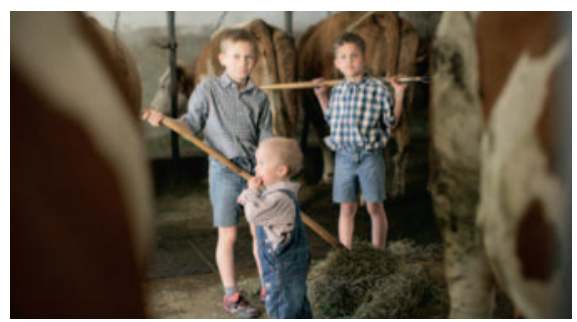

Auch nicht-mikrobielle Stoffe haben präventives Potenzial: Sialinsäure schützt Kinder mit engem Kontakt zu Nutztieren vor Asthma. (CUZH vom Schweizerischen Institut für Allergieund Asthmaforschung der Universität Zürich in Zusammenarbeit mit dem Center for Allergy Research and Education (CK-CARE) in Davos und dem Kinderspital St. Gallen zeigt [1]: «Der frühkindliche Kontakt zu Tieren und auch der Verzehr von tierischen Nahrungsmitteln scheint die Entzündungsreaktionen des Immunsystems zu regulieren», so Immunologe Frei. Seine Studie zeigt, dass die N-Glykolylneuraminsäure (Neu5Gc) dafür verantwortlich ist. Sie kommt in Wirbeltieren - also in vielen Bauernhoftieren - verbreitet vor, fehlt jedoch im menschlichen Organismus.

\section{Antikörper als Maß für Kontakt mit Bauernhoftieren}

Menschen produzieren aufgrund einer genetischen Mutation kein Neu5Gc. Sie können die Sialinsäure aber über Tierkontakt oder auch über den Verzehr von tierischen unverarbeiteten Lebensmitteln aufnehmen und in ihre Glykoproteine einbauen. Der Kontakt mit Neu5Gc löst im Menschen eine Antikörperreaktion aus, die als Maß für den Kontakt mit Bauernhoftieren dienen kann. Die Forscher haben die Konzentrationen von Neu5Gc-Antikörpern in Serum-Proben von Kindern gemessen, die im Rahmen von zwei epidemiologischen Studien (PARSIFALund PASTURE-Studie) gesammelt wurden. Der Vergleich der Neu5Gc-Antikörper-Konzentrationen bei 1235 Kindern und dem Vorkommen von Asthma zeigte eindeutig: «Bauernkinder wiesen viel mehr Antikörper ge- gen Neu5Gc im Blut auf - und Kinder mit mehr Antikörper litten wesentlich seltener an Asthma», so Frei. Die positive Wirkung der Sialinsäure auf die Atemwege wurde am Mausmodell bestätigt: Über die Nahrung aufgenommene Neu5Gc-Moleküle verbesserten die Lungenfunktion der Mäuse und reduzierten somit die Symptome von Asthma.

\section{Vom Bauernhof-Effekt zur Allergieprävention}

Um den Mechanismus zu verstehen, wie Neu5Gc auf das menschliche Immunsystem wirkt, analysierten die Forscher in vitro und in vivo verschiedene Zellen des Immunsystems, die bei einer entzündlichen Reaktion eine Rolle spielen. Demnach reduziert der Kontakt mit Neu5Gc nicht selbst die Immunglobuline $E$, sondern vielmehr wird eine antientzündliche Reaktion des Immunsystems angestoßen, die durch regulatorische T-Zellen vermittelt wird. Diese dämpfen die Fehlreaktionen des Immunsystems und wirken stark antientzündlich. «Unsere Forschungsresultate können wohlmöglich einen wichtigen Grundstein für eine wirksame Allergieprävention legen», fasst Frei zusammen.

\section{Literatur}

1 Frei R, Ferstl R, Roduit C, et al.: Exposure to non-microbial N-glycolylneuraminic acid protects farmers' children against airway inflammation and colitis. J Allergy Clin Immunol 2017;17:pii:S0091-6749(17)30994-6.

Universität Zürich $^{\text {uzH }}$

www.uzh.ch $\rightarrow$ Medienmitteilungen

\section{Universitätsklinikum Frankfurt}

\section{EU-weiter Kampf gegen besondere Lungenkrankheiten}

Das Europäische Referenznetzwerk für seltene Krankheiten des respiratorischen Systems (ERN-LUNG) kann mit seiner Arbeit beginnen: Die Fördervereinbarung mit der Europäischen Kommission wurde Ende Juni unterzeichnet. Das groß angelegte EU-Projekt setzt sich europaweit für die Verbesserung der Vorbeugung, Diagnose und Behandlung von seltenen und/oder komplexen Lungensowie Atemwegserkrankungen ein. Dazu engagieren sich die Projektpartner - ausge- wiesene Experten in ganz Europa - für eine flächendeckende Verbesserung der Patientenversorgung durch Entwicklung von Diagnose- und Therapiestandards sowie Fortbildung und Forschung. «Unser Ziel ist es, Exzellenz bei der Versorgung und Forschung zugunsten der von seltenen Atemwegs- und Lungenerkrankungen betroffenen Patienten zu sichern und zu fördern», sagt Prof. TOF Wagner, Koordinator von ERN-LUNG und Leiter des Zentrums für Seltene Erkrankun- gen am Universitätsklinikum Frankfurt. Insgesamt besteht das Netzwerk derzeit aus 60 Zentren in 12 europäischen Ländern und ist in neun Subnetzen organisiert. Diese Struktur spiegelt die Vielfalt der Krankheiten wider, die das Atmungssystem beeinflussen.

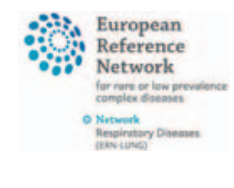

www.kgu.de $\rightarrow$ Aktuelles 
Sammelt sich im Körper z.B. aufgrund eines genetischen Defekts zu viel Eisen an, lagert es sich unter anderem in Leber, Bauchspeicheldrüse, Herz und Gelenken ein und schädigt deren Funktion. Die Lunge war diesbezüglich noch nicht unter Verdacht geraten, doch auch sie ist betroffen, wie Heidelberger Wissenschaftler in einem interdisziplinären Projekt mit Arbeitsgruppen des Deutschen Zentrums für Lungenforschung in Gießen und Hannover nun entdeckt haben [1]. «Im Tiermodell für eine genetisch bedingte und schwer verlaufende Form der Eisenspeicherkrankheit war die Lunge, jedenfalls bestimmte Zelltypen des Lungengewebes, beinahe ebenso mit Eisen überladen wie die Leber», sagt Joana Neves, Abteilung
Onkologie, Hämatologie, Immunologie und Pneumologie am Zentrum für Kinder- und Jugendmedizin Heidelberg. Bei den Mäusen erschwerte dies die Atmung: Die Lunge war weniger dehnbar, das Atemvolumen infolge verringert. Wie es sich beim Menschen verhält, muss noch geprüft werden.

Die aktuellen Ergebnisse legen jedoch nahe, diese Problematik vor allem bei Patienten mit Eisenüberladung und zusätzlich auftretenden Lungenerkrankungen im Blick zu behalten. Denn es häufen sich Hinweise, dass Eiseneinlagerungen auch bei einer Reihe chronischer Lungenerkrankungen wie COPD vorkommen - hier vermutlich durch die anhaltende Entzündung verursacht - und mit einem schwereren
Verlauf einhergehen. Die Forscher vermuten, dass auch die genetisch bedingte Eisenüberladung Lungenprobleme begünstigt oder verschlimmert. Dem sollte man zukünftig mehr Beachtung schenken und die Patienten entsprechend beraten.

\section{Literatur}

1 Neves J, Leitz D, Kraut S, et al.: Disruption of the hepcidin/ferroportin regulatory system causes pulmonary iron overload and restrictive lung disease. EBioMedicine 2017;20:230-239.

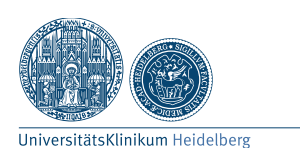

www.uni-heidelberg.de

\section{MeinAllergiePortal}

\section{Experten informieren über allergische Rhinitis und Asthma}

Mit über 1,2 Millionen Lesern pro Jahr ist MeinAllergiePortal (www.mein-allergie-portal.com) eine intensiv genutzte Informationsquelle im Internet, die Patienten, Interessierte und Mediziner gleichermaßen schätzen. Im Fokus stehen Themen wie allergische Rhinitis, Asthma und COPD. Dabei werden Ursachen, Mechanismen, Symptome, Diagnose und Therapieoptionen ausführlich vorgestellt. Um medizinisch korrekte Informationen zu gewährleisten, werden die Informationen in Form von Experteninterviews präsentiert oder stammen direkt aus der Feder von Ärzten.

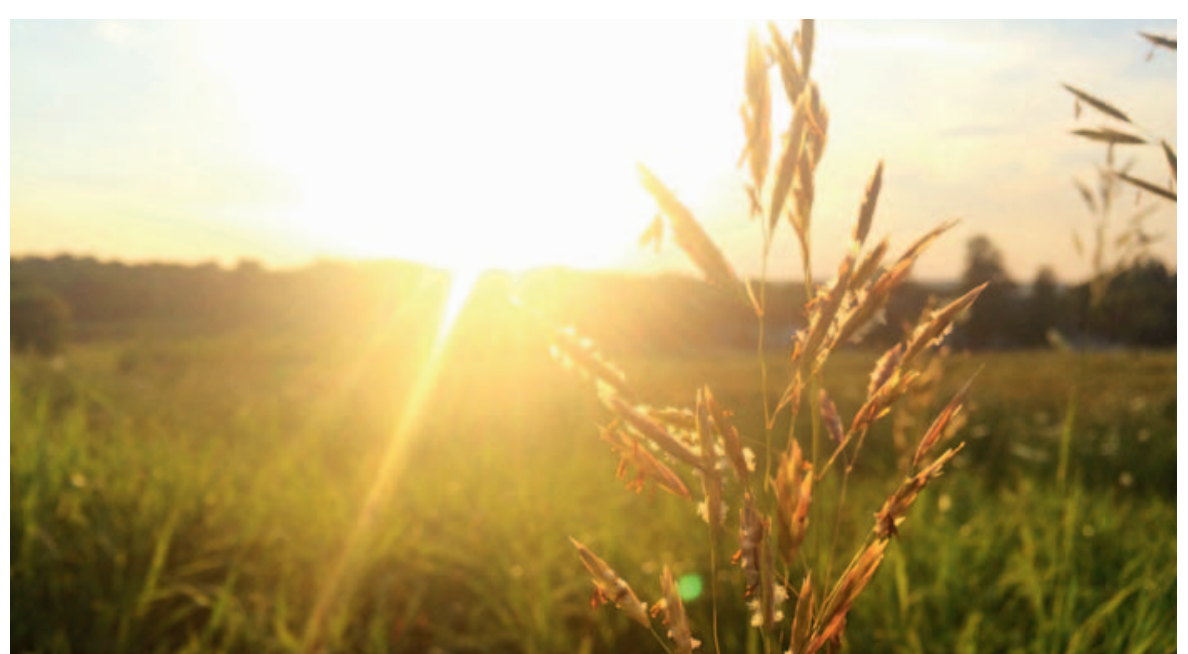

über das Thema «Allergien: Beeinträchtigen sie die schulische Leistung beim Kind?». Auch das Risiko des Etagenwechsels, d.h. der Ausweitung einer allergischen Rhinitis auf die tieferen Atemwege, wird häufig von den Betroffenen nicht gesehen. Im Interview «Etagenwechsel vom Heuschnupfen zum Pollenasthma: Wie lässt sich das verhindern?» mit Prof. Claus Franz Vogelmeier, Universitätsklinikum Marburg, wird die Problematik ausführlich dargestellt. Darüber hinaus stellt MeinAllergiePortal Therapien vor, die den aktuellsten Empfehlungen der Leitlinien entsprechen. Im Interview zum Thema «Hyposensibilisierung: Wie kann sie einen Etagenwechsel verhindern?» erklärt Dr. Peter Kardos, Gemeinschaftspraxis \& Zentrum Pneumologie, Allergologie, Schlafmedizin, Klinik Maingau vom Roten Kreuz in Frankfurt, welche Optionen aktuell zur Behandlung der allergischen Rhinitis zur Verfügung stehen.

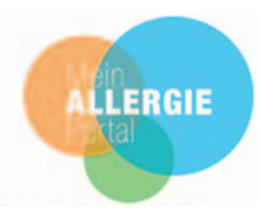

www.mein-allergie-portal.com 
Phthalate, die als Weichmacher in Kunststoffen eingesetzt werden, können das Allergierisiko bei Kindern deutlich erhöhen. Das konnten Forscher des Helmholtz-Zentrums für Umweltforschung (UFZ) gemeinsam mit Wissenschaftlern der Universität Leipzig und des Deutschen Krebsforschungszentrums (DKFZ) in einer gemeinsamen Studie zeigen [1]. Für Kinder besteht demnach ein größeres Risiko, ein allergisches Asthma zu entwickeln, wenn die Mutter während der Schwangerschaft und Stillzeit besonders stark durch Phthalate belastet war. Zu Beginn der Studie untersuchte das UFZ-Forscherteam den Urin von Schwangeren aus der Mutter-Kind-Kohortenstudie LINA (Lebensstil und Umweltfaktoren und deren Einfluss auf das Neugeborenen-Allergierisiko) und fahndete nach Stoffwechselprodukten (Metaboliten) von Phthalaten. Die Höhe der gefundenen Konzentrationen wurde in Bezug zum Auftreten von allergischem Asthma bei den Kindern gesetzt. «Es zeigte sich ein eindeutiger Zusammenhang zwischen erhöhten Konzentrationen des Metaboliten von Butylbenzylphthalat (BBP) im Urin der Mütter und dem Vorkommen von allergischem Asthma bei den Kindern», erklärt Dr. Irina Lehmann, die die LINA-Studie leitet. Die Ergebnisse aus der Mutter-Kind-Kohorte konnten die Forscher in Zusammenarbeit mit Kollegen der Medizinischen Fakultät der Universität Leipzig im Mausmodell bestätigen. Dabei wurden Mäuse während der Schwangerschaft und Stillzeit einer Phthalat-Belastung ausgesetzt, die zu vergleich- baren Urin-Konzentrationen des BBP-Metaboliten führte, wie sie auch bei hochbelasteten Müttern der LINA-Kohorte beobachtet wurden. Die Nachkommen zeigten eine deutliche Neigung zu allergischem Asthma, wobei selbst die Enkelgeneration noch betroffen war. Bei den erwachsenen Mäusen gab es dagegen keine verstärkten Allergiesymptome. «Entscheidend ist also der Zeitpunkt: Ist der Organismus während der frühen Entwicklungsphase Phthalaten ausgesetzt, kann das Auswirkungen auf das Krankheitsrisiko bis in die übernächste Generation haben», erläutert PD Dr. Tobias Polte, Leiter der Forschungsgruppe Experimentelle Allergologie und Immunologie am UFZ. «Durch die Phthalat-Belastung wird also offenbar die pränatale Prägung verändert.»

Epigentische Veränderungen nachgewiesen Bei Mäusen konnten die Forscher nachweisen, dass ein durch DNA-Methylierung ausgeschaltetes Repressor-Gen für die Entstehung des allergischen Asthmas verantwortlich ist. Ob dieser Mechanismus auch beim Menschen eine Rolle spielt, untersuchten die Forscher erneut anhand der LINA-Kohorte, indem sie bei den Kindern mit allergischem Asthma nach dem entsprechenden Gen suchten und den Methylierungsgrad sowie die Genaktivität erfassten. Auch hier zeigte sich, dass das Gen durch Methylgruppen blockiert war und nicht abgelesen werden konnte. «Mithilfe unseres translationalen Studienansatzes vom Menschen über das Mausmodell und

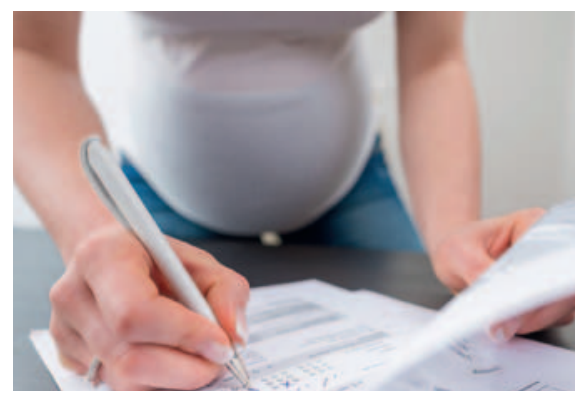

Im Rahmen der Mutter-Kind-Kohorten-Studie LINA untersuchen UFZ-Wissenschaftler Lebensstil und Umweltfaktoren von Schwangeren und deren Einfluss auf das Neugeborenen-Allergierisiko. CUFZ / André Künzelmann.

die Zellkultur wieder zurück zum Menschen - konnten wir zeigen, dass offensichtlich epigenetische Veränderungen dafür verantwortlich sind, dass Kinder bei starker mütterlicher Phthalat-Belastung während der Schwangerschaft und Stillzeit ein erhöhtes Risiko haben, ein allergisches Asthma zu entwickeln», sagt Polte. «Ziel unserer weiteren Forschung wird es sein, zu verstehen, wie genau bestimmte Phthalate eine Methylierung von Genen hervorrufen, die für die Allergieentstehung relevant sind.»

\section{Literatur}

1 Jahreis S, Trump S, Bauer M, et al.: Maternal phthalate exposure promotes allergic airway inflammation over 2 generations through epigenetic modifications. J Allergy Clin Immunol 2017;6:pii:S0091-6749(17)30570-5.

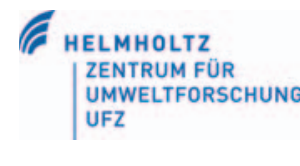

www.ufz.de $\rightarrow$ Medien/Presse

\section{Telemedizin}

\section{Standortunabhängige Versorgung bei Asthma}

Wohnen Patienten mit COPD oder Asthma im ländlichen Gebieten, müssen sie häufig lange Fahrtwege für die lungenfachärzliche Versorgung in Kauf nehmen. Das telemedizinische Projekt «Rheinland-Pfalz atmet durch! Telemedizin für eine gesunde Lunge» soll diesen Patienten die medizinische Versorgung erleichtern. In einem kürzlich gestarteten Pilotprojekt werden 120 lungenkranke Patienten mit der «Asthma
Plus-App» sowie einem tragbaren Lungenmessgerät ausgestattet und durch eine lungenfachärztliche Schwerpunktpraxis in Koblenz betreut. Der behandelnde Arzt kann durch Echtzeiteinblick in die Messdaten zielgenau und schnell reagieren. Das erleichtert Ärzten die Arbeit und ermöglicht Patienten eine standortunabhängige Versorgung. Qurasoft GmbH, ein Start-Up-Unternehmen, das aus der Universität Kob-
lenz-Landau hervorging, hat das Monitoring-System entwickelt und erhält hierfür eine Förderung von 300000 EUR aus Rheinland-Pfälzischen Landesmitteln.

\section{COAsthma Plus hr digitales Asthma Tagebuch}

www.uni-koblenz-landau.de $\rightarrow$ Pressemitteilungen 
Welche Evidenz haben Omega-3-Nahrungsergänzungsmittel auf Patienten mit zystischer Fibrose? Wiederkehrende Zyklen von Infektion und Entzündung führen bei Patienten mit zystischer Fibrose zur Verschlechterung der Lungenfunktion. Studien deuten darauf hin, dass Omega-3-Fettsäuren, die beispielsweise aus Fischölen gewonnen werden, der Entzündung entgegenwirken können und sich damit günstig auf die chronischentzündliche Erkrankung auswirken können. Das Chochrane-Review zu diesem Thema [1] umfasst vier kleine Studien, in denen Omega3-Nahrungsergänzungsmittel mit einem Placebo verglichen werden. Die Evidenz ist auf
Stand vom 13. August 2015. Insgesamt nahmen 91 Kinder und Erwachsene an den Studien teil, bei einer Studiendauer zwischen 6 Wochen und 6 Monaten. Das Hauptergebnis des Reviews belegt, dass eine regelmäßige Nahrungsergänzung mit Omega-3-Fettsäuren Patienten mit zystischer Fibrose einen Nutzen bei wenigen Nebenwirkungen bringen könnte. Es gibt jedoch keine ausreichende Evidenz aus den vier zugrunde liegenden Studien, um eindeutige Schlussfolgerungen zu ziehen oder die routinemäßige Gabe solcher Nahrungsergänzungsmittel bei Patienten mit zystischer Fibrose zu empfehlen. Um den klinischen Nutzen einer Omega-3-Nah- rungsergänzung zu bewerten und die geeignete Dosierung zu bestimmen, sind größere und längere Studien erforderlich.

Auszug aus einer Übersetzung durch S. Schmidt-Wussow, freigegeben durch Cochrane Schweiz.

Literatur

1 Oliver C, Watson H: Omega-3 fatty acids for cystic fibrosis. Cochrane Database Syst Rev 2016;5:CD002201.

\section{(icatme Library}

www.cochranelibrary.com

\section{Klinikum Universität Münster}

\section{Selbstauflösender Bronchial-Stent für Säugling}

Anfang Dezember des letzten Jahres kam ein Mädchen mit einem mehrfachen komplexen Herzfehler auf die Welt. In ihrer Heimat Polen konnten die Ärzte dem Mädchen nicht helfen und schickten es deshalb ans Universitätsklinikum Münster in die Abteilung für Kinderherzchirurgie, wo der Herzfehler Anfang des Jahres erfolgreich operiert wurde. Dennoch konnte der Säugling nach der OP nicht selbständig atmen, weil das Bronchialsystem nach der Herzoperation stark komprimiert war. «Insbesondere der linke Hauptbronchus zwischen Herz und Aorta war zu 90\% verengt», sagt Oberarzt Dr. Claudius Werner, Leiter des Bereichs Pädiatrische Pneumologie. Ein Stent als Platzhalter sollte die Bronchialwege offenhalten. Normalerweise werden bronchiale Stents in Abständen von 6-8 Wochen ge- wechselt. Dazu ist jeweils erneut ein Eingriff nötig, der für die Patienten mit einem deutlichen Risiko von Komplikationen wie Blutungen verbunden ist. Daher entschieden sich die behandelnden Ärzte für ein selbstauflösendes Implantat, das als maßangefertigtes Modell bei einem Spezialanbieter hergestellt wurde. Eigentlich für die Anwendung in der Speiseröhre entwickelt, kann das Implantat auch im Bronchialsystem verwendet werden. Mitte April setzte das Team der Interventionellen Pneumologie am UKM den Stent ein und bereits 5 Wochen nach dem Eingriff konnte das Kind wieder komplett selbstständig atmen. Der Stent in seinen Bronchien sitzt gut, muss aber dennoch möglicherweise nach der Auflösung ersetzt werden. Doch das Komplikationsrisiko ist minimiert, weil er einfach wieder an dieselbe Stelle gesetzt werden kann. Weitere Herzoperationen bleiben dem Mädchen jedoch nicht erspart - zudem müssen ihre Eltern mit ihr regelmäßig zur Nachsorge zu den Experten nach Münster reisen.

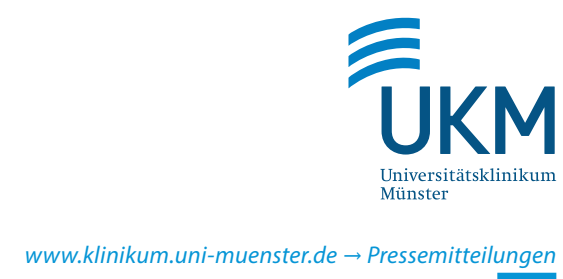

Die Inhalte dieser Rubrik sind von der Redaktion ausgewählt und bearbeitet. Die Auswahl erfolgt unabhängig von der Industrie und in Abstimmung mit dem Herausgeber der Zeitschrift. 